\section{International Scientific Journal Theoretical \& Applied Science}

p-ISSN: 2308-4944 (print) e-ISSN: 2409-0085 (online)

Year: $2018 \quad$ Issue: $03 \quad$ Volume: 59

Published: $30.03 .2018 \quad$ http://T-Science.org
Andrei Mastislavovich Korneev

Doctor of Technical Sciences, Professor, director of the institute of Lipetsk State Technical University, Russia, Lipetsk weenrok@mail.ru

Andrei Vladimirovich Sukhanov postgraduate student, assistant of Lipetsk State Technical University, Russia, Lipetsk pm03sav@mail.ru

Ilya Andreevich Shipulin postgraduate student, assistant of Lipetsk State Technical University, Russia, Lipetsk shipulin-ilya@yandex.ru

\title{
OPTIONS OF ORGANIZATION AND GENERAL SCHEMES OF THE STOCHASTIC OPTIMIZATION ALGORITHM BASED ON THE METHOD OF SIMULATING ANNEALING
}

\footnotetext{
Abstract: The article gives an overview of the existing modifications of the optimization algorithm simulated annealing (simulated annealing) based on the Markov random search in discrete multidimensional spaces. The theoretical foundations for constructing schemes for the stochastic search for a global minimum of the objective function $f: R^{n}$ by the annealing simulation method are formulated. The principles of influence of numerical parameters are described - temperature, energy of the system, boundaries of the search area, the number of iterations, - the rate of convergence of the algorithm and the accuracy of the values determined with its help. The description of three modifications of the random search algorithm simulated annealing (modifications $A, B, C$ ) is given, their advantages and disadvantages are separately highlighted. A block diagram of the complete procedure for finding the minimum of the objective function by the annealing simulation method is presented. A comparative analysis of the Boltzmann scheme and the Cauchy scheme of the organization of the random search algorithm of the global minimum simulated annealing is carried out. Examples of the use of modifications of the random search algorithm simulated annealing in practice are given.

Key words: random search, annealing simulation algorithm, random variable, stop parameter, chemical composition, composite structure, cast iron expansion coefficient, specific heat of cast iron.

Language: Russian

Citation: Korneev AM, Sukhanov AV, Shipulin IA (2018) OPTIONS OF ORGANIZATION AND GENERAL SCHEMES OF THE STOCHASTIC OPTIMIZATION ALGORITHM BASED ON THE METHOD OF SIMULATING ANNEALING. ISJ Theoretical \& Applied Science, 03 (59): 1-5.

Soi: http://s-o-i.org/1.1/TAS-03-59-1 Doi: crossef https://dx.doi.org/10.15863/TAS.2018.03.59.1

\section{ВАРИАНТЫ ОРГАНИЗЦИИ И ОБЩИЕ СХЕМЫ АЛГОРИТМА СТОХАСТИЧЕСКОЙ ОПТИМИЗАЦИИ НА ОСНОВЕ МЕТОДА ИМИТАЦИИ ОТЖИГА}

Аннотация: В статье приводится обзор существующих модификаџий алгоритма оптимизации simulated annealing (имитация отжига) на основе марковского случайного поиска в дискретных многомерных пространствах. Сформулированы теоретические основы для построения схем стохастического поиска глобального минимума целевой функции $f: R^{n}$ методом имитации отжжга. Описаны принциипь влияния численных параметров, - температура, энергия системы, границы области поиска, количество итераций, - на скорость сходимости алгоритма и точность определяемых с его помощью значений. Приведено описание трёх модификаций алгоритма случайного поиска simиlated annealing (модификации A, Б, В), отдельно выделены их преимущества и недостатки. Представлена блоксхема полной процедуры поиска минимума иелевой функичии методом имитации отжжга. Проведен сравнительный анализ схемы Больцмана и схемы Коши организации алгоритма случайного поиска глобального минимума simulated annealing. Приведены примеры использования модификаций алгоритма случайного поиска simulated anпеaling на практике.
} 
Ключевые слова: случайный поиск, алгоритм имитации отжига, случайная величина, параметр остановки, ческий состав, композитная структура, коэффициент литейного расширения чугуна, удельная теплоемкость чугуна.

\section{Introduction}

По результатам исследований, проведенных С. Киркпатриком, предложен метод случайного поиска $[1,6]$, - simulated annealing (имитация отжига), - глобального минимума функции в условиях наличия явных ограничений на варьируемые параметры $[2,3,6,7,8]$. Метод simulated annealing предназначен для нахождения глобального минимума функции $f(x)$, заданной для $x$ из некоторого дискретного или непрерывного пространства $\Omega$. Элементы этого множества $\Omega$ являются состояниями некоторой воображаемой физической системы, а значения функции $f(x)$ рассматриваются в качестве энергии системы $E=f(x)$. В каждый момент предполагается заданной параметр системы $T$ (температура), который, как правило, уменьшается с течением времени. После нахождения в состоянии $x$ при параметре со значением $T$, последующее состояние системы выбирается посредством заданным порождающим семейством вероятностных распределений $G(x, T)$, которое задает случайный элемент со значениями в пространстве $\Omega$ при фиксированных $x$ и $T$. После генерации нового состояния система $x^{\prime}=G(x, T)$, система переходит к новому шагу в состояние $x^{\prime}$ с вероятностью $p(\Delta E, T), \quad$ в противном случае продолжается процесс генерации нового значения $x^{\prime} . \Delta E$ определяется здесь как приращение функции энергии системы, а величина $p(\Delta E, T)$ является вероятностью принятия нового состояния.

\section{Materials and Methods}

Поиск глобального минимума целевой функции $f: R^{n}$ осуществляется в некоторой собственной области подмножества метрического пространства $R^{n}$ :

$$
f\left(x_{1}, \ldots, x_{n}\right) \rightarrow \min , x \in \Omega, \Omega \in R^{n},
$$

где подмножество $\quad \Omega \quad$ определяется ограничениями в виде $q(x)=0, q: R^{n}$.

Пусть некоторое множество $X=\left\{x_{1}, \ldots, x_{n}\right\}$ принадлежит подмножеству $\Omega \in R^{n}$, на котором определена скалярная функция $f(x)$. Функция $f(x)$ в точке $x^{*}$ имеет локальный минимум, если в некоторой $\varepsilon$-окрестности этой точки справедливо следующее условие: $f\left(x^{*}\right)<f(x),\left|x-x^{*}\right| \leq \varepsilon$.

В качестве вероятности перехода поиска в новое состояние $p(\Delta E, T)$ выбирается либо точное значение величины

$$
p(\Delta E, T)=\frac{1}{1+\exp \left(\frac{\Delta E}{T}\right)}
$$

либо его приближенное значение

$$
p(\Delta E, T)=\exp \left(-\frac{\Delta E}{T}\right)
$$

Наиболее часто используется формула (3). При этом для случая $\Delta E<0$ будет справедливо следующее: $p(\Delta E, T)<1, \quad$ и тогда вероятность перехода в новое состояние $p(\Delta E, T)$ считается равной 1. Поиск минимума целевой функции заканчивается при достижении параметром температуры $T$ некоторого заданного значения $T_{\text {end. }}$ Отсюда следует, что схема метода отжига задается следующими параметрами:

- закон уменьшения (изменения) параметра $T(k)$, где $k$ - номер шага;

- порождающее семейство вероятностных распределений $G(x, T)$;

- функция вероятности перехода системы в новое состояние $p(\Delta E, T)$.

Наиболее простой вариант алгоритма метода стохастического поиска simulated annealing описывается следующим образом:

1. Случайным образом выбирается начальная точка $x=x_{0}, x_{0} \in S$. Определяется текущее значение функции энергии системы $E=f(x)$.

2. Пока не выполнено условие остановки поиска $T(k)<T_{\text {end }}$, выполнять следующий шаг $k$ ой итерации основного цикла:

2.1. Сравниваются значения функции $E$ в состоянии $x$ и в состоянии, являющемся на текущем моменте глобальным минимумом. В случае если значение $E=f(x)$ оказалось меньше, то изменить значение глобального минимума. $G(x, T)$.

2.2. Генерируется новое состояние $x^{\prime}=$

2.3. Определяется значение функции в новом состоянии $E^{\prime}=f\left(x^{\prime}\right)$.

2.4. Генерируется случайное число $\alpha \in[0,1]$.

2.5. Для случая, когда $\alpha<p\left(E^{\prime}-E, T(k)\right)$ установить $x \leftarrow x^{\prime}, E \leftarrow E^{\prime}$ и перейти итерации $k+1$. Если $\alpha \geq p\left(E^{\prime}-E, T(k)\right)$ повторять шаг 2.2 до тех пор, пока не будет найдена подходящая точка $x^{\prime}$.

Возможны следующие модификации приведенного алгоритма:

Модификация алгоритма simulated annealing А. На шаге 2.5 в случае если точка $x^{\prime}$ не является подходящей переход к следующей итерации возможен при условии, что следующая итерация начинается с точки $x$ с новым значением уменьшающегося параметра $T$. 


\begin{tabular}{l|lr|ll|ll} 
& ISRA (India) & $=\mathbf{1 . 3 4 4}$ & SIS (USA) & $=\mathbf{0 . 9 1 2}$ & ICV (Poland) & $=\mathbf{6 . 6 3 0}$ \\
Impact Factor: & ISI (Dubai, UAE) $=\mathbf{0 . 8 2 9}$ & PUHЦ (Russia) $=\mathbf{0 . 2 0 7}$ & PIF (India) & $=\mathbf{1 . 9 4 0}$ \\
& GIF (Australia) & $\mathbf{0 . 5 6 4}$ & ESJI (KZ) & $=4.102$ & IBI (India) & $=\mathbf{4 . 2 6 0}$ \\
& JIF & $=\mathbf{1 . 5 0 0}$ & SJIF (Morocco) & $=\mathbf{2 . 0 3 1}$ & & \\
\hline
\end{tabular}

Модификация алгоритма simulated annealing Б. Для случая большой размерности $S$ с целью ускорения работы алгоритма в качестве оценки точки глобального минимума принимается последнее значение $x$. Однако такой подход может привести к худшему решению, особенно, если значение параметра $T$ значительно превышает ноль к моменту завершения работы алгоритма.

Модификация алгоритма simulated annealing B. В начале работы алгоритма на шаге 1 принимается $x^{\prime} \leftarrow x_{0}$, а на шаге 2.2 новая точка $x^{\prime}$ вычисляется рекуррентно по формуле $x^{\prime}=G\left(x^{\prime}\right.$, $T(k))$. Данный подход препятствует «застреванию» алгоритма в окрестности определенной точки. Тут стоит отметить, что при такой реализации алгоритм имитации отжига практически не отличается от обычного стохастического поиска, что приводит к потере ряда преимуществ алгоритма.

Блок-схема полной процедуры поиска минимума целевой функции методом имитации отжига представлен на рисунке 1.

Наиболее часто в различных модификациях алгоритма имитации отжига новая точка $x_{k+1}^{\prime}$ определяется на основе текущей точки $x$ посредством применения к ней оператора, который случайным образом модифицирует соответствующую точку. Результатом применения оператора становится новая точка $x^{*}$. Точка $x^{*}$ принимается в качестве новой точки $x^{\prime}{ }_{k+1}$ с вероятностью $P\left(\mathrm{x}^{*} \rightarrow x_{k+1}^{\prime} \mid x_{k}^{\prime}\right)$, которая вычисляется в соответствии с распределением Гиббса:

$$
P\left(x^{*} \rightarrow x_{k+1}^{\prime} \mid x_{k}^{\prime}\right)=\left\{\begin{array}{cc}
1, & f\left(x^{*}\right)-f\left(x_{k}\right)<0, \\
\exp \left(-\frac{f\left(x^{*}\right)-f\left(x_{k}\right)}{Q_{k}}\right), & f\left(x^{*}\right)-f\left(x_{k}\right) \geq 0 .
\end{array}\right.
$$

Здесь $Q_{k}>0$ - элементы убывающей положительной сходящейся последовательности, закон и скорость убывания которой задаются. Последовательность $Q_{k}$ в значительной степени предопределяет точность результата стохастического поиска минимума целевой функции $f(x)$. 


\begin{tabular}{|c|c|c|c|c|c|c|}
\hline Impact Factor: & $\begin{array}{l}\text { ISRA (India) } \\
\text { ISI (Dubai, UAE } \\
\text { GIF (Australia) } \\
\text { JIF }\end{array}$ & $\begin{array}{r}=1.344 \\
=0.829 \\
=0.564 \\
=1.500\end{array}$ & $\begin{array}{l}\text { SIS (USA) } \\
\text { PИНЦ (Russia) } \\
\text { ESJI (KZ) } \\
\text { SJIF (Morocco) }\end{array}$ & $\begin{array}{l}=0.912 \\
=0.207 \\
=4.102 \\
=2.031\end{array}$ & $\begin{array}{l}\text { ICV (Poland) } \\
\text { PIF (India) } \\
\text { IBI (India) }\end{array}$ & $\begin{array}{l}=6.630 \\
=1.940 \\
=4.260\end{array}$ \\
\hline
\end{tabular}

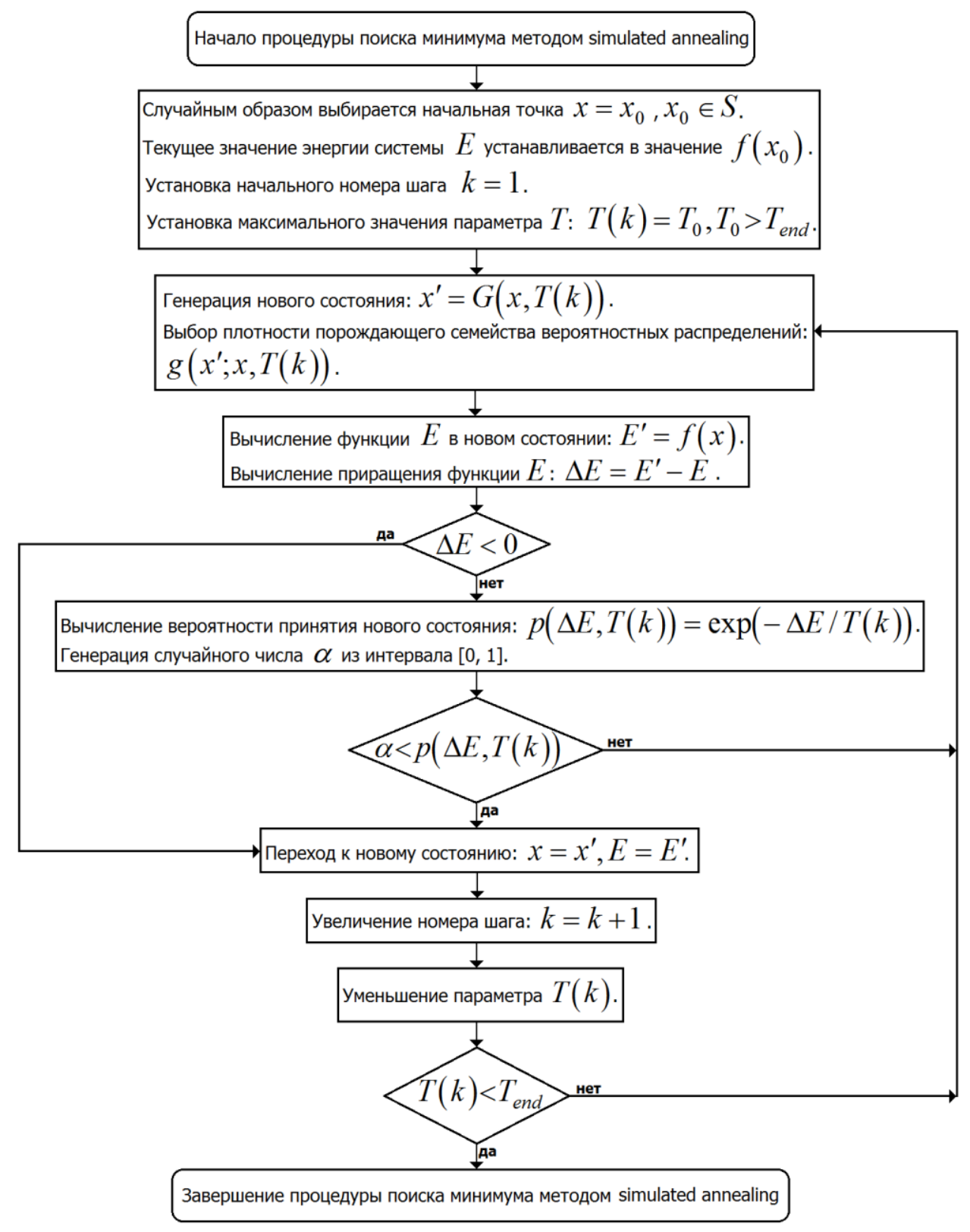

Рисунок 1 - Блок-схема процедуры поиска минимума целевой функции методом simulated annealing.

Алгоритм имитации отжига (simulated annealing) сходен с методом градиентного спуска, однако по причине того, что новые точки выбираются случайным образом, они будут попадать в локальные минимумы реже, чем при поиске методом градиентного спуска. Алгоритм имитации отжига не гарантирует нахождения глобального минимума функции. Тем не менее, в случае удачной и правильной генерации новых случайных точек в пространстве $X$ возможно улучшение начального приближения.

Одной из самых распространенных схем метода имитации отжига является схема Больцмана (Больцмановский отжиг). Метрополис Н. использовал данную схему при решении задач статистической физики [4], а С. Киркпатрик для определения оптимальных конфигураций микросхем [1]. В данной схеме изменение параметра температуры $T$ алгоритма задается формулой:

$$
T(k)=\frac{T_{0}}{\ln (1+k)}, k>0 .
$$

Плотность вероятностных распределений выбирается как семейство нормальных распределений с математическим ожиданием $x$ и дисперсией $T$ :

$$
g\left(x^{\prime} ; x, T\right)=\frac{1}{(2 \pi T)^{D / 2}} \cdot \exp \left(-\frac{\left|x^{\prime}-x\right|^{2}}{2 T}\right),
$$


где $D$ - размерность метрического пространства состояний. Доказано, что при больших значениях параметра $T_{0}$ и общем количестве шагов $K$ для больцмановской схемы алгоритма такое семейство распределений гарантирует нахождение глобального минимума. Основной недостаток данной схемы - медленное убывание параметра $T$ (для решений некоторых задач исходное значение $T_{0}$ необходимо уменьшить в 40 раз, что требует не менее $2,35 \cdot 10^{17}$ итераций). По этой причине исследователями Хартли и Цу $[5,9,10]$ была предложена схема алгоритма, в которой для изменения параметра $T$ используется формула $T(k)=T_{0} / k$. При данной схеме не происходит потеря гарантии нахождения минимума за счет использования соответствующим способом нормированных распределений Коши с плотностью

$$
g\left(x^{\prime} ; x, T\right)=\frac{1}{\pi^{(D+1) / 2}} \cdot \frac{T}{\left(\left|x^{\prime}-x\right|^{2}+T^{2}\right)^{(D+1) / 2}}
$$

Иной вид будет иметь распределение плотности для случая одномерного пространства:

$$
g\left(x^{\prime} ; x, T\right)=\frac{1}{\pi} \cdot \frac{T}{\left|x^{\prime}-x\right|^{2}+T^{2}}
$$

Моделирование распределения в пространстве с размерностью $D>1$ производится перемножением $D$ одномерных распределений Коши:

$$
g\left(x^{\prime} ; x, T\right)=\frac{1}{\pi^{D}} \prod_{i=1}^{D} \frac{T}{\left|x^{\prime}-x\right|^{2}+T^{2}}
$$

В данной схеме глобальный минимум определяется гарантированно только при изменении параметра $T$ со скоростью, не превышающей

$$
T(k)=\frac{T}{k^{1 / D}}
$$

что намного медленнее Больцмановской схемы.

\section{Conclusion}

Из приведённого обзора численных методов оптимизации, основанных на метаэвристических алгоритмах случайного поиска глобального минимума, можно сделать вывод, что до настоящего времени не существует разработанных подходов к выбору схемы поиска для решения конкретной задачи. В частности, для определения оптимальных значений численных коэффициентов при решении задач оптимизации химического состава или структуры чугунных сплавов необходимо моделирование с выбором модификаций и с использованием нескольких различных схем, после чего, произведя анализ результатов их работы, сделать выводы о приемлемости разработанных алгоритмов для решения данной задачи.

\section{References:}

1. Kirkpatrick S., Gelatt C. D., Vecchi M. P. (1983) Optimization by simulated annealing // Science. 1983. Vol. 220. P. 671-680.

2. Lopatin A.S. (2005) Metod otzhiga // Stokhasticheskaya optimizatsiya $\mathrm{v}$ informatike. SPb.: Izd-vo SPbGU, 2005. Vyp. 1. p. 133-149.

3. Ingber L. (1993) Simulated Annealing: Practice versus theory // Mathematical and Computer Modelling. 1993. Vol. 18(11). P. 29-57.

4. Metropolis N., Rosenbluth A. W., Rosenbluth M. N., Teller A. H., and Teller E. (1953) Equation of State Calculations by Fast Computer Machines // J. Chemical Physics. 21. 6. June. 1953. P. 1087-1092.

5. Szu H. H., Hartley R. L. (1987) Fast Simulated Annealing // Physical Letters A. 122. 1987. P. 157-162.
6. Tikhomirov A., Stojunina T., Nekrutkin V. (2007) Monotonous random search on a torus: Integral upper bounds for the complexity // J. Statistical Planning and Inference. 2007. Vol. 137, No. 12. P. 4031-4047.

7. Spall J.C. (2003) Introduction to stochastic search and optimization: estimation, simulation, and control. New Jersey: Wiley, 2003. 618 p.

8. Yao X. (1995) A New Simulated Annealing Algorithm // International Journal of Computer Mathematics. 56. 1995. P. 161-168.

9. Yin G. (1999) Rates of convergence for a class of global stochastic optimization algorithms // SIAM Journal on Optimization. 1999. Vol. 10. No. 1. P. 99-120.

10. Zhigljavsky A., Zilinskas A. (2008) Stochastic Global Optimization. Berlin: Springer, 2008. $262 \mathrm{p}$. 